\title{
A Novel Alternative for the Treatment of Diabetic Foot Wounds: A Three Dimensional Porous Dermal Matrix
}

\author{
Evren H. Gokce' ${ }^{1}$, Sakine Tuncay Tanrıverdi ${ }^{1}$, Ipek Eroglu ${ }^{2}$, Nicolas Tsapis $^{3}$, \\ Goksel Gokce ${ }^{4}$, Elias Fattal ${ }^{3}$, Ozgen Ozer ${ }^{1}$ \\ ${ }^{1}$ Department of Pharmaceutical Technology, Faculty of Pharmacy, Ege University \\ 35100, Bornova, Izmir, Turkey \\ evrenhomangokce@gmail.com; sakinetuncay@windowslive.com \\ ${ }^{2}$ Department of Basic Pharmaceutical Sciences, Faculty of Pharmacy, Hacettepe University \\ 06100 Ankara, Turkey \\ ${ }^{3}$ Institut Galien Paris-Sud, CNRS, Univ. Paris-Sud, Université Paris-Saclay \\ 92296 Châtenay-Malabry, France \\ ${ }^{4}$ Department of Pharmacology, Faculty of Pharmacy, Ege University \\ 35100, Bornova, Izmir, Turkey
}

\section{Extended Abstract}

Diabetic wounds tend to heal slowly and the healing process can be very complicated due to polymicrobial infection and heavy exudate formation which place patients at a higher risk for limb amputation [1]. The investigations in wound care field revealed bioactive dermal matrices produced from a variety of proteins of extracellular matrix (ECM) have the ability to promote the healing process [2]. In addition reduction/termination of the persistent inflammation and elimination of free radicals by the introduction of an antioxidant could be an important strategy to improve healing [3]. Therefore a novel alternative for the treatment of diabetic foot wounds consisting of a three dimensional collagen-laminin porous dermal matrix impregnated with resveratrol (RSV)-loaded hyaluronic acid (HA) and dipalmitoylphosphatidylcholine (DPPC) microparticles was evaluated. Characterization, in vitro release, microbiological, ex vivo and in vivo studies were performed. Spherical microparticles of $30.2 \pm 0.3 \mu \mathrm{m}$ were obtained with a RSV encapsulation efficacy of $98.7 \%$. Scanning electron microscopy (SEM) and confocal laser scanning microscopy showed that particles were well dispersed in the dermal matrix from the surface to deeper layers. Collagenase degraded dermal matrix, however the addition of RSV loaded microparticles delayed the degradation time. The release of RSV was sustained and reached $70 \%$ after 6 h. Histological changes and antioxidant parameters in different treatment groups were investigated in full-thickness excision diabetic rat model. The highest healing score was obtained with the dermal matrix impregnated with RSV-microparticles with an increased antioxidant activity. Collagen-laminin dermal matrix with RSV microparticles can be an effective and safe option for the treatment of diabetic wounds requiring long recovery.

\section{Aknowledgements}

This work was supported by TUBITAK 1001 [No:111S183] and Institut Galien Paris-Sud is a member of the Laboratory of Excellence LERMIT supported by a grant from ANR (ANR-10-LABX-33).

\section{References}

[1] P. G. Bowler, B. I. Duerden and D. G. Armstrong, "Wound microbiology and associated approaches to wound management," Clin. Microbiol. Rev. vol. 14, pp. 244-269, 2001.

[2] Y. K. Bashmakov, S. Assaad-Khalil and I. M. Petyaev, "Resveratrol may be beneficial in treatment of diabetic foot syndrome," Med. Hypotheses, vol. 77, pp. 364-367, 2011.

[3] V. Kant, A. Gopal, N.N. Pathak, P. Kumar, S. K. Tandan and D. Kumar, "Antioxidant and anti-inflammatory potential of curcumin accelerated the cutaneous wound healing in streptozotocin-induced diabetic rats," Int. Immunopharmacol., vol. 20, pp. 322-330, 2014. 